\section{BS53 THE ROLE OF PLASMA MEMBRANE CALCIUM ATPASE 4 (PMCA4) IN VASCULAR REMODELLING DURING ABDOMINAL AORTIC ANEURYSM FORMATION}

${ }^{1}$ Kinza Khan, ${ }^{2}$ Miguel R Campanero, ${ }^{3}$ James Cotton, ${ }^{4} J u a n$ Miguel Redondo, ${ }^{1}$ Angel L Armesilla*. ${ }^{1}$ RIHS, FSE, University of Wolverhampton; ${ }^{2}$ Instituto de Investigaciones Biomedicas Alberto Sols, CSIC-UAM, Madrid, Spain; ${ }^{3}$ Department of Cardiology, Heart and Lung Centre, New Cross Hospital, Wolverhampton, UK; ${ }^{4}$ Centro Nacional de Investigaciones Cardiovasculares, Madrid, Spain

\subsection{6/heartjnl-2019-BCS.214}

Introduction An Abdominal Aortic Aneurysm (AAA) is a permanent localised dilation of the abdominal aorta, classified by a diameter of $3.0 \mathrm{~cm}$. Predominant in men aged $>65$ years, this chronic degenerative disease accounts for 6,000 deaths per year in the UK. Inflammatory infiltration and enzymatic destruction of the elastic lamellae and extracellular matrix (ECM) proteins constitute the key histopathological features. An imbalance in the expression of ECM remodelling proteins, such as matrix metalloproteinases and their inhibitors, contributes to the progressive weakening of the vascular wall. AAAs are also associated with modulation and apoptosis of vascular smooth muscle cells (VSMCs). Understanding the molecular mechanisms behind this remodelling process, may identify potential novel candidates for therapeutic intervention.

Plasma Membrane Calcium ATPase 4 (PMCA4) belongs to a family of transmembrane receptors that extrude calcium from the cytosol to the extracellular environment. There are four isoforms (PMCA1-4) encoded by the ATP2B1-4 genes. PMCA4 has been identified as a negative regulator of VEGFinduced angiogenesis in our laboratory. Vascular remodelling during aneurysm formation includes increased angiogenesis alongside the above-mentioned extracellular matrix degradation and chronic inflammation. With PMCA1 and PMCA4 being the major isoforms expressed in aortic tissue, we aim to determine whether they have a role during pathological remodelling of aneurysmal disease.

Methods Cell culture, qRT-PCR, Western Blot, siRNA-mediated gene silencing

Results Here we examined the role of IL1Beta on the expression of PMCA4 using qRT-PCR. We show a time- and dosedependent downregulation of ATP2B4 mRNA expression in human primary Aortic Endothelial Cells (AoEC). This translated to a reduction in protein levels, confirmed using western blot studies. To determine the functional consequences of reduced PMCA4 expression, we screened an array of genes related to the extracellular matrix. si-RNA mediated knockdown of PMCA4 in the presence of an inflammatory stimulus, led to a 3.27 -fold and 4.37-fold enhancement on the IL1Betainduced expression of the metalloproteinase ADAMTS1 $(\mathrm{P}=$ $0.025915)$ and the cell adhesion molecule SELP $(\mathrm{P}=$ 0.007897), respectively.

Conclusion AAA current treatment involves surveillance until the risk of rupture outweighs the risk of surgery. A complete understanding of the molecular and cellular mechanisms implicated in aortic dilation and AAA progression will aid the development of therapeutic strategies. Our results demonstrate that inflammatory stimuli reduce the levels of PMCA4 in AoEC. Reduced expression of PMCA4 leads to increased expression of proteins related to ECM remodelling, suggesting a role for PMCA4 during AAA progression.

Conflict of interest None

\section{BS54 VARIATION IN CARDIAC LONG NON-CODING RNAS IN CONGENITAL HEART DISEASE}

${ }^{1}$ Stephanie Baross*, 'Simon Williams, "Kathryn Hentges, ${ }^{1}$ Andrew Sharrocks, ${ }^{2}$ Bernard Keavney. ${ }^{1}$ University of Manchester; ${ }^{2}$ Faculty of Biology, Medicine and Health, University of Manchester

\subsection{6/heartjnl-2019-BCS.215}

Congenital heart disease (CHD) is the most common birth defect affecting approximately 1\% of live births. CHD shows a high degree of heritability but the genetic causes are still poorly understood. Many of the known causative genes of $\mathrm{CHD}$ are those with roles in gene regulation, such as transcription factors and histone modifiers. One possible explanation of the "missing heritability" of CHD is long non-coding RNAs (lncRNAs), which often act as regulators of gene expression and which have been excluded from previous exome-focussed studies. Previous in vivo and in vitro work has identified six lncRNAs with roles in regulation of heart development (CARMEN, EINCR1, FENDRR, METEOR, STX18-AS1, and UPPERHAND). However, these lncRNAs have not been studied in CHD patients to determine if they have a causal role in CHD. We have used whole genome sequencing data from $607 \mathrm{CHD}$ patients included in the 100,000 Genomes Project to identify variants in these lncRNAs. Variants present in CHD patients in these lncRNAs were filtered against variants in a control set of 68,221 genomes to remove common variants present in the wider population. The control set contains whole genome sequences from gnomAD (genome aggregation database) and individuals from the 100,000 Genomes Project who do not have CHD or related conditions such as syndromes which include CHD (Kabuki syndrome and RASopathies) or other conditions with overlapping aetiologies (aortopathies and neurodevelopmental disorders). Variants in all six tested lncRNAs were significantly enriched $(\mathrm{p}<0.0001)$ in CHD patients compared to the control group, with between 8 and 10 times more variants seen per individual, indicating variants in cardiac lncRNAs may contribute to $\mathrm{CHD}$. The identified $\mathrm{CHD}$-unique variants have been cross-referenced with eQTL and predicted secondary structures to prioritise variants which are likely to affect the function of the IncRNA. Where available, parental DNA is used to identify de novo variants in CHD patients with unaffected parents and variants which co-segregate with incidence of CHD in families. In future, prioritised variants will be modelled in suitable in vitro models to measure the effects of the variants on lncRNA function and heart development pathways. This work will allow the contribution of these cardiac lncRNAs to CHD to be studied for the first time.

Conflict of interest None

\section{BS55 REGULATION OF SKELETAL MUSCLE STEM CELL FUNCTION IN A MOUSE MODEL OF EXPERIMENTAL ATHEROSCLEROSIS AND HYPERLIPIDAEMIA}

Antonios Matsakas*, Joseph Barlow, Peggy Sfyri, Sandrine Verpoorten, David Scully. Hull York Medical School

\subsection{6/heartjnl-2019-BCS.216}

Introduction Apolipoprotein E deficient (ApoE KO) mice are an established model of hyperlipidemia and atherosclerosis, 